\title{
Contribuições dos profissionais de saúde frente ao trauma peniano
}

\author{
Contributions of health professionals to penile trauma \\ Contribuciones de los profesionales de la salud al trauma del pene
}

Rayssa Stéfani Sousa Alves ORCID: https://orcid.org/0000-0002-9666-675X Pontifícia Universidade Católica de Goiás, Brasil E-mail: rayssastefani02@gmail.com Mariana Pereira Barbosa Silva ORCID: https://orcid.org/0000-0003-0852-8099 Universidade Estadual do Piauí, Brasil E-mail: marianapbsilvaa@gmail.com Airton César Leite ORCID: https://orcid.org/0000-0001-7184-8488

Centro Universitário Santo Agostinho, Brasil E-mail: ainton.cesar2014@gmail.com Ana Graziela Soares Rêgo Lobão

ORCID: https://orcid.org/0000-0003-3003-954X Centro Universitário Uninovafapi, Brasil

Centro Universitário UniFacid Wyden, Brasil E-mail: grazielarego@hotmail.com Francisca Edinária de Sousa Borges ORCID: https://orcid.org/0000-0002-6023-8059 Universidade Estadual do Piauí, Brasil E-mail: edinariasousa@hotmail.com

Viviane Silva de Moura ORCID: https://orcid.org/0000-0003-3043-3854 Centro Universitário UniFacid Wyden, Brasil E-mail: vivisilva_21@hotmail.com Yulle Morais Gomes

ORCID: https://orcid.org/0000-0003-0715-2106

Centro Universitário UniFacid Wyden, Brasil E-mail: yullemorais100@hotmail.com Natana Maranhão Noleto da Fonseca ORCID: https://orcid.org/0000-0003-4592-3004

Centro Universitário Uninovafapi, Brasil E-mail: natana_fonseca1@hotmail.com

Maria Clara Osório Meneses Carvalho ORCID: https://orcid.org/0000-0003-2603-5159

Centro Universitário UniFacid Wyden, Brasil

E-mail: mcosorio97@yahoo.com.br

Mariana Magalhães Bergantini Zanovello ORCID: https://orcid.org/0000-0002-2932-9219

Centro Universitário UniFacid Wyden, Brasil

E-mail: mariana_zanovello@hotmail.com

Poliana Zanotto Manoel

ORCID: https://orcid.org/0000-0003-3162-5292

Faculdade Evangélica Mackenzie Paraná, Brasil

E-mail: poliana_zm@hotmail.com Agnes Zanotto Manoel

ORCID: https://orcid.org/0000-0003-4488-3127

Faculdade Evangélica Mackenzie Paraná, Brasil E-mail: agneszmx@gmail.com Aedna Canuto de Sousa Rolim ORCID: https://orcid.org/0000-0003-3245-9142

Faculdade Pernambucana de Saúde, Brasil E-mail: aedna.canuto@gmail.com Jessica Reis Lopes

ORCID: https://orcid.org/0000-0001-9029-6068 Centro Universitário Tocantinense Presidente Antônio Carlos, Brasil

E-mail: jessica123reis2017@gmail.com

Luciana Angelica Gonçalves

ORCID: https://orcid.org/0000-0003-1427-2763

Universidade Federal de Rondonópolis, Brasil E-mail: luciana.angelicca@gmail.com

Samara Atanielly Rocha

ORCID: https://orcid.org/0000-0002-5622-9280

Faculdade de Saúde e Humanidades Ibituruna, Brasil

E-mail: samaraatanielly@ outlook.com 


\begin{abstract}
Resumo
Este estudo tem como objetivo identificar os fatores que levam a ocorrência do trauma peniano durante a penetração no intercurso sexual, e compreender as contribuições dos profissionais de saúde ao paciente com fratura de pênis. Trata - se de um estudo descritivo, de abordagem qualitativa, realizado entre os meses de maio a dezembro de 2020, mediante a revisão sistemática de artigos indexados na Plataforma da Biblioteca Virtual da Saúde (BVS). Os critérios de inclusão foram, artigos completos na linguagem portuguesa, inglesa e espanhola. Publicados na íntegra de acordo com a temática proposta, e artigos científicos publicados nas bases de dados compreendendo os anos de 2000 a 2020. Como critérios de exclusão, não foram considerados artigos que não tivessem relevância com a temática, materiais duplicados, incompletos, resumos, resenhas, debates e materiais indisponíveis na íntegra. Para o levantamento dos dados foram analisados 10 artigos científicos. Os resultados do estudo descrevem que a fratura ocorre quando o pênis ereto sai da cavidade vaginal e ao tentar penetrar novamente, o pênis é dobrado de maneira brusca e forçada. Seguindo das diversas contribuições dos profissionais de saúde frente à este incidente. Concluímos que o trauma peniano é considerado uma emergência urológica rara, compreendendo um impacto significativo no ponto de vista geral e na saúde sexual do indivíduo, a lesão peniana deve ser analisada com base na anamnese, e no exame físico do indivíduo, métodos de diagnóstico por imagem devem ser solicitados frente a incerteza e precisão diagnostica.
\end{abstract}

Palavras-chave: Ferimentos e lesões; Genitália masculina; Pênis.

\begin{abstract}
This study aims to identify the factors that lead to the occurrence of penile trauma during penetration during sexual intercourse, and to understand the contributions of health professionals to patients with fractured penises. It is a descriptive study, with a qualitative approach, carried out between the months of May and December 2020, through the systematic review of articles indexed in the Platform of the Virtual Health Library (VHL). Inclusion criteria were full articles in Portuguese, English and Spanish. Published in full according to the proposed theme, and scientific articles published in the databases covering the years 2000 to 2020. As exclusion criteria, articles that were not relevant to the theme, duplicate, incomplete materials, abstracts, reviews, debates and materials unavailable in full. To collect the data, 10 scientific articles were analyzed. The results of the study describe that the fracture occurs when the erect penis leaves the vaginal cavity and when trying to penetrate again, the penis is bent abruptly and forced. Following the various contributions of health professionals to this incident. We conclude that penile trauma is considered a rare urological emergency, comprising a significant impact on the individual's general point of view and sexual health, penile injury should be analyzed based on anamnesis, and on the individual's physical examination, diagnostic methods by image must be requested in view of uncertainty and diagnostic accuracy.
\end{abstract}

Keywords: Wounds and injuries; Male genitalia; Penis.

\title{
Resumen
}

Este estudio tiene como objetivo identificar los factores que conducen a la ocurrencia de traumatismo peneano durante la penetración durante las relaciones sexuales, y comprender las contribuciones de los profesionales de la salud a los pacientes con fractura de pene. Se trata de un estudio descriptivo, con enfoque cualitativo, realizado entre los meses de mayo y diciembre de 2020, mediante la revisión sistemática de artículos indexados en la Plataforma de la Biblioteca Virtual en Salud (BVS). Los criterios de inclusión fueron artículos completos en portugués, inglés y español. Publicados íntegramente según la temática propuesta, y artículos científicos publicados en las bases de datos de los años 2000 a 2020. Como criterios de exclusión, artículos no relevantes para la temática, duplicados, materiales incompletos, resúmenes, revisiones, debates y materiales no disponibles en su totalidad. Para la recogida de datos se analizaron 10 artículos científicos. Los resultados del estudio describen que la fractura se produce cuando el pene erecto sale de la cavidad vaginal y al intentar penetrar nuevamente, el pene se dobla de forma brusca y forzada. Tras las distintas aportaciones de los profesionales sanitarios a este incidente. Concluimos que el traumatismo de pene se considera una urgencia urológica poco común, que tiene un impacto significativo en el punto de vista general y la salud sexual del individuo, la lesión del pene debe analizarse con base en la anamnesis y en el examen físico del individuo, métodos de diagnóstico por La imagen debe solicitarse en vista de la incertidumbre y la precisión del diagnóstico.

Palabras clave: Heridas y lesiones; Genitales masculinos; Pene.

\section{Introdução}

A fratura de pênis (FP) corresponde a ruptura da membrana albugínea quando o pênis está em condição ereta. Trata se, de uma eventualidade incomum, com incidência de 1 em 175 homens, de idade média de 28 anos. Este acontecimento implica na necessidade de uma abordagem profissional adequada, visto que tal evento provoca uma significativa repressão social (Amer, 2016). 
Para Alves (2004), a fratura de pênis trata - se de um trauma peniano fechado, compreendendo a ruptura da túnica albugínea, consistindo em uma camada de tecido fibroso que envolve os corpos cavernosos, onde um dos principais invólucros do pênis se apresenta posicionado abaixo da fáscia de Buck. Essa ruptura ocorre geralmente durante a atividade sexual, quando sua espessura é reduzida em até $75 \%$, tornando-o mais frágil. O evento é resultante ao aumento da pressão sob a albugínea consistindo no quadro do trauma,

A denominação fratura de pênis, não consiste em uma fratura propriamente dita, visto que, o pênis não é um órgão ósseo. Portanto, o retardamento na intervenção desse agravo, pode resultar em uma perda significativa da capacidade sexual por vários anos (Amer, 2016).

Geralmente, essa lesão ocorre quando o pênis ereto é golpeado contra o períneo no intercurso sexual, ou durante a masturbação (Castro, 2009). A túnica albugínea compreende uma das fáscias mais resistentes do corpo humano, sendo assim, o risco de fratura peniana aumenta durante a ereção, em decorrência da pressão conforme sua estrutura, estiramento e afinamento, reduzindo sua espessura em até 75\% (Carvalho, 2013; Andrade, 2015).

Portanto, os mecanismos que envolvem a ocorrência do trauma peniano são variáveis que compreendem aspectos culturais. A título de exemplo, é comum em alguns países, o hábito de estalar o pênis para alcançar a detumescência. A fratura de pênis tem manifestação clínica típica. O indivíduo relata ter escutado o som (estalido) provocado pela ruptura do corpo cavernoso, seguido por dor, detumescência instantânea, hematoma, e edema, causando a deformação do pênis (Eke, 2015).

Segundo os estudos de Castro (2009) e Mahapatra (2015), o diagnóstico de fratura de pênis é alicerçado na história clínica do paciente, seguido da execução do exame físico. A suspeita de fratura peniana ocorre quando o paciente relata a ocorrência repentina de estalido durante atividade sexual seguido de edema e descoloração peniana, resultando em deformidade física, assemelhando - se, ao formato de berinjela.

Fundamentado nos estudos da European Association of Urology (EAU), Carvalho (2013) e Garofalo et al. (2015), afirmam que, se houver incerteza no diagnóstico da ocorrência do trauma peniano, deve - se, confirmar mediante métodos de imagem, como: ultrassonografia, ressonância magnética e cavernosografia, para localizar detalhadamente a lesão.

Carvalho (2013) e Garofalo et al. (2015) consideram a ultrassom como o método menos invasivo, portanto a constatação diagnostica depende de uma visualização eficaz, que por sua vez apresenta - se prejudicada pelo edema e hematoma, ficando em desvantagem para a ressonância, que é um método de mais eficiência, porém, menos acessível.

Já a cavernosografia é um exame realizado para avaliar a drenagem venosa e a anatomia dos corpos cavernosos do pênis. Este método pode apresentar resultados falso-negativos, e o retardamento da realização deste, pode ocasionar graves reações alérgicas devido ao contraste infundido, além de outras complicações (Carvalho, 2013; Garofalo et al., 2015).

Ainda nos estudos de Carvalho (2013) e Garofalo et al. (2015), mediante a ocorrência da fratura peniana, existe cerca de 10 a $20 \%$ de chances de ocorrer ruptura uretral. Esta condição deve ser investigada quando houver sinais de uretrorragia, hematúria macroscópica, além de sintomas miccionais e retenção urinária, podendo ser confirmado através da uretrocistografia retrógrada, cistoscopia flexível e exames de maior precisão.

Segundo Cunha, Melo e Maroclo (2000), o trauma peniano é caracterizado pela perda de segmentos de pele, ruptura peniana, contusão testicular, e amputações. Esta eventualidade divide - se, em trauma durante a penetração no intercurso sexual (45\%), contusão peniana (45\%), queimaduras e acidentes industriais (10\%).

Sendo assim, quais são os fatores que levam a ocorrência do trauma de pênis? E quais são as contribuições assistenciais do enfermeiro frente ao paciente vítima de trauma peniano? A importância desse estudo consiste em aprimorar o conhecimento acerca da temática proposta; identificar quais são as estratégias assistenciais do enfermeiro frente aos cuidados ao paciente vítima de trauma peniano, e contribuir de forma a complementar nas bases de dados cientificas. 
Este estudo tem como objetivo identificar e compreender os fatores que levam a ocorrência do trauma peniano durante a penetração no intercurso sexual heterossexual, e evidenciar as contribuições assistenciais dos profissionais de saúde ao paciente com trauma peniano.

\section{Metodologia}

Trata-se de um estudo descritivo, de abordagem qualitativa, segundo Gil (2008), este tipo de estudo busca descrever as características de determinadas populações ou fenômenos.

A análise dos dados foi realizado entre os meses de maio a dezembro de 2020, mediante a revisão sistemática de artigos indexados e publicados na Plataforma da Biblioteca Virtual da Saúde (BVS), que compreende as bases de dados SciELO (Scientific Eletronic Library Online), LILACS (Literatura Latino-Americana e do Caribe em Ciências da Saúde), MEDLINE (Literatura Internacional em Ciências da Saúde), BDENF (Base de Dados de Enfermagem).

Foram usados os seguintes descritores em base DeCS (Descritores em Ciências da Saúde): Ferimentos e Lesões; Genitália Masculina; Pênis, Combinados com o operador booleando “AND” entre os descritores citados.

Os critérios de inclusão para a seleção dos conteúdos foram, artigos completos na linguagem portuguesa, inglesa e espanhola. Publicados na íntegra de acordo com a temática proposta, e artigos científicos publicados nos referidos bancos de dados compreendendo os anos de 2000 a 2020. Como critérios de exclusão, não foram considerados artigos que não tivessem relevância com a temática, materiais duplicados, incompletos, resumos, resenhas, debates, relato de caso, relato de experiência, publicados em anais de eventos e materiais indisponíveis na íntegra.

\section{Resultados e Discussão}

A partir da revisão de literatura e análise dos estudos indexados nas bases de dados eletrônicas, acerca da temática proposta, foram encontrados 62 estudos científicos, sendo que, apenas 28 estudos foram selecionados, 22 atenderam aos critérios de inclusão previamente estabelecidos, destes, 12 foram excluídos com base nos critérios de exclusão, restando 10 artigos para composição e análise do estudo. O fluxograma com o detalhamento das etapas de pesquisa está apresentado a seguir na Figura 1. 
Figura 1. Fluxograma de identificação e seleção dos artigos. 2020.

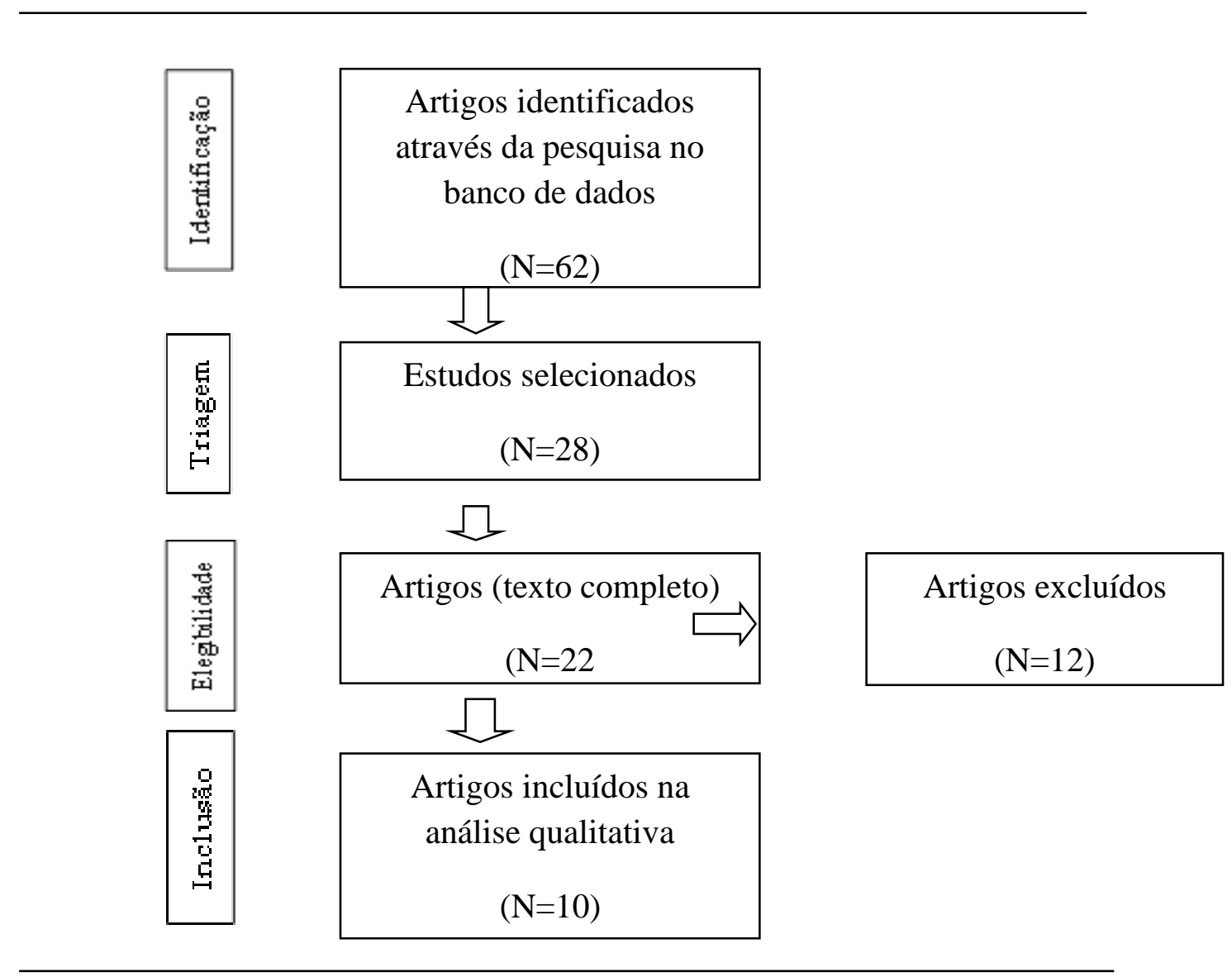

Fonte: Autores (2020).

Para análise, interpretação, discussão e elaboração dos dados, foram explorados 09 estudos compreendidos entre os anos de 2000 a 2020. Apresentando os fatores que levam a ocorrência do trauma peniano em atividades sexuais heterossexuais, e a assistência dos profissionais de saúde frente à este evento.

Para Napier (2019) compreender a anatomia do pênis e a complexidade de seus planos fasciais, é fundamental para analisar com precisão a gravidade da fratura peniana, pois essas camadas influenciam o padrão de extravasamento de hematoma após o incidente.

Os invólucros fasciais do pênis podem ser categorizadas em três camadas distintas, sendo elas, tecido conjuntivo fibroso, facial profundo e superficial. A camada fibrosa nomeada túnica albugínea, é compreendida como uma bainha fibroelástica densa que contorna os corpos cavernosos do pênis, sendo fundida na linha média entre eles. Essa camada incorpora um composto por fibras de sentido longitudinal que se liga aos ramos pubianos, consistindo em uma camada circular que compõe o septo entre as cavernas. É considerada a fáscia mais resistente do corpo e a mais importante na definição de uma fratura peniana (Napier, 2019).

Segundo Barros et al. (2018), os principais fatores de risco para a ocorrência da fratura peniana compreende evidências histológicas de um processo inflamatório crônico contido na túnica albugínea de indivíduos com trauma peniano, em que tecido cicatricial fibroso e inelástico da lesão anterior enfraqueceria o corpo cavernoso, tornando-o mais fraco e vulnerável para um novo evento de fratura. Essa teoria é comprovada pela predominância de casos de fratura de pênis que se 
iteram no corpo cavernoso ipsilateral. Portanto, também há indícios de que o tecido cicatricial poderia provocar uma distribuição desigual de tensão na túnica albugínea, resultando em ruptura do lado contralateral.

De acordo com Carlos et al. (2013), na América do Norte a relação sexual compreende a principal causa do trauma peniano. Na maioria das vezes, a fratura ocorre quando a mulher está posicionada em cima do homem durante a atividade sexual, e o pênis sai da cavidade vaginal. Ao tentar penetrar novamente, o pênis ereto é galopeado contra o quadril ou períneo da parceira, fazendo com que o pênis dobre de maneira brusca e forçada. Em posições sexuais que o homem fica por cima ou atrás da mulher, os riscos diminuem significativamente.

Em países do Oriente Médio, uma das principais causas do trauma peniano compreende a prática da automanipulação (Taghaandan), método executado que resulta no dobramento do pênis ereto para alcançar um rápido retorno do pênis para seu estado flácido após a ereção, ou seja, estado de detumescência (Carlos et al., 2013).

Geralmente, a fratura ocorre na região proximal da haste peniana. A laceração da albugínea é unilateral e transversa, localizada no segmento ventral, distal ao ligamento suspensório do pênis (Alves, 2004).

Segundo os estudos de Alves (2004) na maioria das vezes, a lesão ocorre na base do pênis, possibilitando a saída extensa da pressão sanguínea concentrada no pênis ereto. Imediatamente ocorre a perda do estado de ereção, manifestando aspectos de hematoma e deformação peniana. Na condição do rompimento ascender o corpo esponjoso que envolve a uretra, cerca de 10 a $20 \%$ dos traumas penianos desenvolvem consequências como, a uretrorragia ou a hematúria microscópica.

Durante a inspeção do exame físico do pênis após a ocorrência do trauma, podemos evidenciar deformidade em formato de berinjela, com presença de edema, hematoma e desvio peniano para o lado oposto ao da fratura. Na presença de sinais e sintomas urinários, deve - se considerar a hipótese de lesão uretral concomitante, podendo ocorre em até $20 \%$ dos casos (Ishikawa et al., 2003).

Os profissionais de saúde são fundamentais no processo de constatação diagnóstica do trauma peniano, sendo executado com base na história clínica e exame físico do indivíduo (Carvalho, 2013). Métodos como a ultrassonografia, ressonância magnética e a cavernosografia são solicitadas pelos profissionais frente a incerteza do diagnóstico (Garofalo et al., 2015).

O reparo cirúrgico é uma das contribuições dos profissionais de saúde, sendo considerado o método mais eficaz para tratamento do trauma peniano e correção da uretra, caso seja constatado a coexistência das duas lesões, quando comparado ao tratamento conservador consistindo em imobilização compressiva, medicações fibrinolíticas, antibióticos e inibidores da ereção (Garofalo et al., 2015).

O cirurgião é responsável pela escolha do procedimento conforme sua experiência, podendo ser uma incisão por circuncisão com desenluvamento da superfície peniana ou uma incisão longitudinal sobre a região afetada, seguindo por sutura dos locais apresentando perda de continuidade tecidual. As orientações dos profissionais de saúde incluem abstinência sexual por aproximadamente 6 semanas (Ory \& Bailly, 2019).

Outras contribuições dos profissionais de saúde compreendem a realização do curativo compressivo e em alguns casos a inserção do cateter vesical de demora, após a cirurgia para reparação do trauma peniano (Ibarguren et al., 2006).

A primeira execução cirúrgica da fratura peniana foi descrita por Fetter e Gartman no ano de 1936, desde então, este método de reparo é compreendido como padrão-ouro para o tratamento desse transtorno (Mahapatra et al., 2015; Garofalo M, et al., 2015).

O procedimento cirúrgico está associado ao menor tempo de permanecia no ambiente hospitalar, menores gastos monetários, e satisfação do indivíduo frente a um melhor resultado quanto a reparação da lesão, capacidade mictória e redução da incidência de disfunção erétil (Garofalo M, et al., 2015). 
Andrade (2015), afirma que, o reparo cirúrgico compreende a drenagem do hematoma, contenção da hemorragia, sutura dos corpos cavernosos e da uretra, caso esteja lesionada. Os autores Djakovic et al. (2005), Alves (2004) e Muentener et al. (2004) consideram que, o tratamento conservador deve ser sustentado apenas em situações de objeção do paciente à cirurgia; comparecimento tardio aos serviços de saúde devido ao constrangimento; fraturas e hematomas pequenos ou rupturas mínimas dos corpos cavernosos.

Segundo Carvalho (2013) e Garofalo et al. (2015), a busca imediata pelos Serviços de Saúde apresentam resultados mais favoráveis em relação às buscas tardias. Estudos evidenciaram que, pacientes submetidos ao procedimento cirúrgico tardio podem desenvolver sequelas, sendo: curvatura homolateral à lesão, diminuição da qualidade da ereção e formação de calo ósseo (Muentener, et al., 2004).

Carlos et al. (2013) consideram em seus estudos que uma das vantagens do procedimento cirúrgico imediato, compreende as baixas taxas de curvatura peniana em índices menores que 5\%. Já o tratamento conservador apresenta taxas maiores que $10 \%$, além da possibilidade de apresentarem abscessos ou placas de fibrose extenuantes em 25 a $30 \%$.

\section{Considerações Finais}

Este estudo demonstrou que a lesão peniana deve ser analisada com base na história clínica, e no exame físico do indivíduo. Considerando a possibilidade de lesão uretral e riscos de possíveis sequelas.

Ainda que, o trauma peniano seja considerado uma emergência urológica rara, compreende - se um impacto significativo no ponto de vista geral e na saúde sexual do indivíduo, considerando que a vítima de ferimento peniano busque intervenção cirúrgica tardia e tenha em média 28 anos de idade.

Assim, uma das atribuições dos profissionais de saúde consiste em conscientizar a população jovem quanto a importância do tratamento imediato, orientando quanto aos sinais e sintomas mais frequentes para facilitar na definição da ocorrência de lesão peniana, sendo: descoloração, edema, desvio peniano, e deformidade em formato de berinjela. Já o sangramento, os sintomas miccionais e a retenção urinária, estão entre os possíveis sinais que indicam ruptura uretral. Os métodos de diagnóstico por imagem devem ser solicitados frente a incerteza e precisão diagnostica.

Outra contribuição dos profissionais de saúde consiste no encaminhamento imediato do indivíduo para a realização do procedimento cirúrgico, visto que, o manejo cirúrgico apresenta melhores resultados de reparação e satisfação da vítima.

\section{Referências}

Alves, L. S. (2004). Fratura de Pênis. Revista do Colégio Brasileiro de Cirurgia. 31(5): 284-286.

Amer, T. (2016). Penile Fracture: A Meta-Analysis. Urologia Internationalis. 96(3): 315-329.

Andrade, M. F., et al. (2015). Abordagem Tardia do Trauma de Pênis: Relato de Caso. Revista Ciências em Saúde; 5 (2).

Barros, R., et al. (2018). Penile refracture: a preliminary report. International braz j urol. 44(4), 800-804.

Carlos, et al. (2013). Fratura peniana - Uma revisão. Revista Eletrônica da Comissão de Ensino e Treinamento da SBU. 2013 (1)2: 15-20.

Carvalho, J., Arlindo, M. (2013). Fratura de Pênis com Trauma de Uretra. Revista do Colégio Brasileiro de Cirurgiões; 40(4): 351-353.

Castro, P. R. (2009). Fratura Peniana: Diagnóstico e Tratamento. Revista Médica de Minas Gerais. 19(2):123-126.

Djakovic, N., et al. (2005). Diretrizes para o Trauma Urológico. European Association of Urology; 47(1): 1-15.

Garofalo, M., et al. (2015). Sex-Related Penile Fracture with Complete Urethral Rupture: A Case Report and Review of The Literature. Archivio Italiano di Urologia e Andrologia. 87(3):260-1.

Ibarguren, R. L., et al. (2006). Penile Fracture with Associated Urethral Rupture. Archivos Espanoles de Urologia. 59 (7):732-736.

Mahapatra, R. S., et al. (2015). Penile Fracture: Our Experience in a Tertiary Care Hospital. The World Journal of Men's Health. 33 (2): $95-102$. 
Research, Society and Development, v. 10, n. 1, e24310111209, 2021 (CC BY 4.0) | ISSN 2525-3409 | DOI: http://dx.doi.org/10.33448/rsd-v10i1.11209

Muentener, M., et al. (2004). Long-Term Experience with Surgical and Conservative Treatment of Penile Fracture. The Journal of Urology. 172:576-9.

Napier, D. (2019). The role of ultrasound in the diagnosis of penile fracture. Sonography; 6: 15 -23.

Ory, J. Bailly, G. (2019). Management of penile fracture. Can Urol Assoc J. 13 (6) 4:72-74.

Sabharwal, S., et al. (2015). Hidden Penile Fracture: An Unusual Presentation and Review of Literature. Urology Annals. 7 (2): 248.

Shukla, A. K., et al. Role of ultrasonography in grading of penile fractures. J Clin Diagn Res. 2015; 9(4): 1-3.

Ishikawa, T., Fujisawa, M., Tamada, H., Inoue, T., Shimatani, N. (2003). Fracture of the penis: nine cases with evaluation of reported cases in Japan. Int $J$ Urol. 10:257-60. 